\title{
The profile of thyroid disorders in patients attending a tertiary care hospital in Pokhara, Nepal
}

\author{
Dr Tirthalal Upadhyaya ${ }^{1} \&$ Dr Raju Sapkota ${ }^{2}$ \\ ${ }^{1}$ Department of Medicine, Gandaki Medical College and Teaching Hospital, Pokhara, Nepal \\ ${ }^{2}$ Vision \& Eye Research Institute, School of Medicine, Faculty of Health, Education and Medical Science, \\ Anglia Ruskin University, East Road, Cambridge, CB1 1PT, UK
}

\begin{abstract}
Background: The profile of thyroid disorder has been reported with limited evidence in Nepal. The aim of this study was to examine the profile and incidence of different types of thyroid disorder in patients attending a hospital appointment in Western Nepal and identify factors associated with it.

Method: This was a hospital-based study. A total of 1000 patients attending the department of medicine at Gandaki Medical College, Teaching Hospital and Diabetes, Thyroid and Endocrinology Care Centre, Pokhara for thyroid examination were recruited. Thyroid disorders were classified as hypothyroidism, hyperthyroidism, T4-thyrotoxicosis including their sub types where applicable. Results: Nearly $33 \%$ of the participants were found to have some form of thyroid disorders. Majority of the patients with thyroid disorder were females (87\%). Hypothyroidism was found to be the most common type of thyroid disorder (57.5\%) followed by hyperthyroidism (37.5\%) and secondary hyperthyroidism (5.0\%). BMI was found to be independently associated with hyperthyroidism on regression model. Conclusion: Hypothyroidism was found to be the most common thyroid disorder followed by hyperthyroidism. The findings highlight that thyroid disorder is a significant clinical burden and a major public health concern in Western Nepal. The findings also emphasize a need to roll out thyroid screening programmes in the general population so that this condition can be identified and treated early.
\end{abstract}

Key Words: Hypothyroidism, Hyperthyroidism, Thyroid Disorder

\section{INTRODUCTION}

It is a widely known fact that thyroid gland produces two hormones, namely triiodothyronine (T3) and thyroxine (T4) ${ }^{1}$, which are essential for our health and wellbeing; they play a vital role in growth, neuronal development, reproduction, and regulation of energy metabolism in our body ${ }^{2}$. The activities of our thyroid gland is regulated by Thyroid Stimulating Hormone (TSH) which is produced by the anterior pituitary gland. British Thyroid Foundation ${ }^{3}$ has outlined the normal levels of these thyroid hormones as following; TSH 0.44.0 milliunits per litre, FT4 9.0-25.0 picomoles per litre, FT3 3.5-7.8 picomoles per litre. However, the units and cut off criteria vary widely between

$\overline{\text { Corresponding author: Dr Tirthalal Upadhyaya, Associate }}$ Professor, Department of Medicine, Gandaki Medical College and Teaching Hospital, Pokhara, Nepal, E mail : tirtha77@, gmail.com different countries and clinical practices.

Hypothyroidism and hyperthyroidism are the two most common types of thyroid disorders reported in the literature ${ }^{4,5}$. In hypothyroidism, the thyroid gland is underactive, as a consequence of which it cannot produce enough hormones. Usually increased TSH level and decreased T4/T3level indicates that thyroid gland may be under-active. Hypothyroidism is often caused by the Hashimoto's disease ${ }^{6}$, which is also called chronic autoimmune lymphocytic thyroiditis. Fatigue, memory problems, constipation, depression, weight gain, weakness, slow heart rate are some of the common symptoms associated with hypothyroidism. In contrary, in hyperthyroidism the thyroid gland is overactive and produces excessive hormones; increased $\mathrm{T} 4 /$ $\mathrm{T} 3$ and decreased TSH levels indicate that thyroid gland may be overactive. Graves' disease is the 
most common cause of hyperthyroidism ${ }^{7}$, affecting about $70 \%$ of people with an overactive thyroid gland. Restlessness, nervousness, racing heart, irritability, increased sweating, shaking, anxiety, trouble sleeping are some of the common symptoms associated with hyperthyroidism.

Other types of thyroid-related disorders include T4 thyrotoxicosis (increased level of T4 hormone, TSH of normal level), sub-clinical hyperthyroidism (decreased TSH level, T4/T3 of normal level), sub-clinical hypothyroidism (increased TSH level, T4/T3 normal level), secondary hypothyroidism (decreased TSH, T4, T3 hormone levels), and secondary hyperthyroidism (decreased TSH, T4, T3 levels).

Prevalence of thyroid disorder is a much debated research topic. Inconsistency across studies in the definition of thyroid disorder, participant selection criteria, environmental and geographical factors, and different techniques and criteria used for assessment of thyroid functions are some of the major factors identified to contribute towards this debate ${ }^{7}$.This is further complicated by social stigmas and lack of awareness associated with thyroid disorders due to which people may feel hesitant to visit a doctor specially in countries where health literacy rate is lower ${ }^{8}$. It is possible that prevalence of thyroid disorder in the literature is often underestimated.

A recent study from Australia reported the prevalence of $0.3 \%$ for both clinical and subclinical hyperthyroidismin general population'. In 2002, United States National Health and Nutrition Examination Survey (NHANES III) found the prevalence of hyperthyroidism and subclinical hyperthyroidism in general population to be $0.5 \%$ and $0.7 \%$, respectively ${ }^{10}$. A meta-analysis of studies from European countries showed the mean prevalence rate of $0.75 \%$ for males and females combined and an incidence rate of 51 cases of thyroid disorder per 100,000 per year $^{11}$. A UK-based longitudinal study found an incidence of thyroid disorder of 80 cases per 100,000 women per year ${ }^{12}$.
Also, a higher prevalence of hyperthyroidism has been found in iodine-deficient countries ${ }^{13}$.

In Nepal the profile of thyroid disorder has been reported with limited evidence. A recent study by Gupta et $\mathrm{al}^{14}$ found the prevalence of thyroid disorder in Achham district hospital to be $17.11 \%$ (range, 14\%-o 20\%), of which hypothyroidism was most common followed by hyperthyroidism. Also females were found to disproportionately have higher prevalence of thyroid disorder compared to men ( $14.7 \%$ vs. $2.4 \%)$. Another study from Eastern Nepal showed that chronic iodine deficiency persisted in a small fraction of pregnant women, and that the mild thyroid dysfunction was also common in these women ${ }^{15}$. Khatiwada et a ${ }^{16}$ reported that thyroid dysfunction was widely common co-morbid condition (38.6\%) in patients with chronic kidney disease. Another study showed that $3.2 \%$ of the school age children have thyroid dysfunction in Eastern Nepal ${ }^{17}$. To our knowledge there are no studies from Western Nepal that have examined the profile of thyroid disorders, which this study examined. In addition to examining the profile, we also fitted regression models to identify which factors (age, gender, BMI, pulse, blood pressure) were associated with thyroid disorder independently.

\section{METHODS}

Study type: This was a hospital-based retrospective study of case-control design. Most of the data were collected at the outpatient department of Gandaki Medical College and Teaching Hospital, Pokhara The study protocol was approved by the Institutional Review Board of the same institute.

Inclusion criteria: (i). Patients attending the Outpatient Department of Gandaki Medical College and Teaching Hospital and Diabetes, Thyroid and Endocrine Care Centre to be evaluated for the thyroid function tests or thyroid disorders; (ii). Patients able to provide informed consent for taking part in the study

Exclusion criteria: (i). Patients not wanting their 
The profile of thyroid disorders in patients attending a...

Jour of Diab and Endo Assoc of Nepal 2019; 3 (2): (42-48)

ISSN Print 2594-3367ＩSSN Online 2631-2107

medical records to be included in the study; (ii). Patients with incomplete data.

In total, 1000 patients examined from May 2016 to April 2018 were recruited retrospectively. Using our previous (unpublished) data, and to attain a power of $80 \%$ with a precision error and type $1(\alpha)$ error of $5 \%$ each, the required sample size was calculated to be 965 . A slightly greater number $(n=1000)$ has been used in this study.

Patients were briefed during their visit that their data could be used to report in scientific journals but they will remain anonymous. All data were handled with applicable ethical guidelines, and followed the tenants of Helsinki Declaration. The reporting follow the Strengthening the Reporting of Observational studies in Epidemiology (STROBE) guidelines ${ }^{18}$.

The patient data were collected by reviewing their medical files by the same author (TU), who is also the consultant endocrinologist at, both, the Gandaki Medical College and Teaching Hospital, and the Diabetes, Thyroid, and Endocrinology Care Centre, Pokhara Nepal. The main variables collected were age, T3, T4, TSH, random blood sugar, systolic and diastolic blood pressure, pulse rate and Body Mass Index (BMI) for each participant. Lab tests were done at either one or the other of the two institutes with which the first author (TU) is affiliated to by certified professionals. Data entry was double checked before processing and analyses.FT3 level of 2.27-4.55 picomoles/litre, FT4 level of 0.65-1.74 picomole/litre and TSH level of 0.355.29 millinternational units/litre were considered normal levels. The following criteria were used to classify thyroid disorders: $\mathrm{t} 3, \mathrm{t} 4$ normal and high level of TSH-subclinical hypothyroidism; low TSH with normal t3,t4 levels-subclinical hyperthyroidism; high $\mathrm{t} 4$ but normal TSH and $\mathrm{t} 3$ levels- thyrotoxicosis; high t4,t3 and TSH levelssecondary hyperthyroidism; low $\mathrm{t} 3, \mathrm{t} 4$, and TSHsecondary hypothyroidism; low $\mathrm{t} 3, \mathrm{t} 4$ but high TSH levels-primary hypothyroidism.
Data were analysed using Statistical Package for the Social Sciences (SPSS, version 24). No data were excluded from the analyses. Data of continuous variables were normally distributed (Levene's test, $p>0.05$ ). Independent $t$-tests was used to compare between any two variables between the participant groups (i.e., with thyroid disorder vs. normal). Multiple linear regression models were fitted to identify which of the variables (age, BP, BMI, pulse, etc.) were independently associated with different thyroid disorders.

\section{RESULTS}

Mean, standard deviation and standard error for participants with some form of thyroid disorder and normal participants is provided in Table 1 . Out of the total 1000 participants, 339 (33.9\%) had some form of thyroid disorder. As expected participant groups (normal vs. thyroid disorder) differed significantly in age, T3, T4, TSH and pulse levels $(p \leq 0.02)$. Surprisingly, diastolic blood pressure (but not the systolic blood pressure) was also found to differ significantly between the participant groups. Since the aim of the study was to examine the profile of patients with thyroid disorder, the subsequent analyses do not include data from normal participants. 
The profile of thyroid disorders in patients attending a...

Jour of Diab and Endo Assoc of Nepal 2019; 3 (2): (42-48)

ISSN Print 2594-3367 ISSN Online 2631-2107

Table 1. Mean, standard deviation, and standard error for the variables examined for participants with and without thyroid disorders. Comparisons between participant groups were done using independent samples t-tests. * indicates significant p-values.

\begin{tabular}{|c|c|c|c|c|c|}
\hline Variable & Participant group & Mean value & $\begin{array}{l}\text { Standard } \\
\text { Deviation }\end{array}$ & $\begin{array}{c}\text { Standard } \\
\text { Error Mean }\end{array}$ & p-values \\
\hline \multirow{2}{*}{ Age (years) } & With thyroid disorder & 42.93 & 11.89 & 0.68 & \\
\hline & Normals & 44.72 & 12.26 & 0.50 & $0.02 *$ \\
\hline \multirow{2}{*}{ T3 (pg/ml) } & With thyroid disorder & 3.20 & 1.94 & 0.12 & \\
\hline & Normals & 2.95 & 0.41 & 0.02 & $<0.001 *$ \\
\hline \multirow{2}{*}{ T4 (ng/dl) } & With thyroid disorder & 3.83 & 5.58 & 0.34 & \\
\hline & Normals & 1.19 & 0.33 & 0.01 & $<0.001 *$ \\
\hline \multirow{2}{*}{ TSH (mlu/ml) } & With thyroid disorder & 8.07 & 9.75 & 0.46 & \\
\hline & Normals & 2.67 & 1.39 & 0.06 & $<0.001 *$ \\
\hline \multirow{2}{*}{ FBS (mg/DL) } & With thyroid disorder & 105.51 & 36.05 & 2.16 & \\
\hline & Normals & 106.05 & 36.86 & 1.52 & 0.97 \\
\hline \multirow{2}{*}{ BP:systole } & With thyroid disorder & 119.17 & 15.69 & 0.89 & \\
\hline & Normals & 119.92 & 16.40 & 0.68 & 0.4 \\
\hline \multirow{2}{*}{ BP:Diastole } & With thyroid disorder & 77.11 & 10.87 & 0.61 & \\
\hline & Normals & 78.49 & 9.69 & 0.40 & $0.04 *$ \\
\hline \multirow{2}{*}{ Pulse/minute } & With thyroid disorder & 79.46 & 8.05 & 0.46 & \\
\hline & Normals & 78.35 & 7.70 & 0.32 & $0.009 *$ \\
\hline \multirow{2}{*}{$\mathrm{BMI}\left(\mathrm{Kg} / \mathrm{m}^{2}\right.$} & With thyroid disorder & 27.17 & 4.95 & 0.28 & \\
\hline & Normals & 27.12 & 4.71 & 0.19 & 0.97 \\
\hline
\end{tabular}

Fig. 1 provides a pie chart showing the incidence of each type of thyroid disorders.

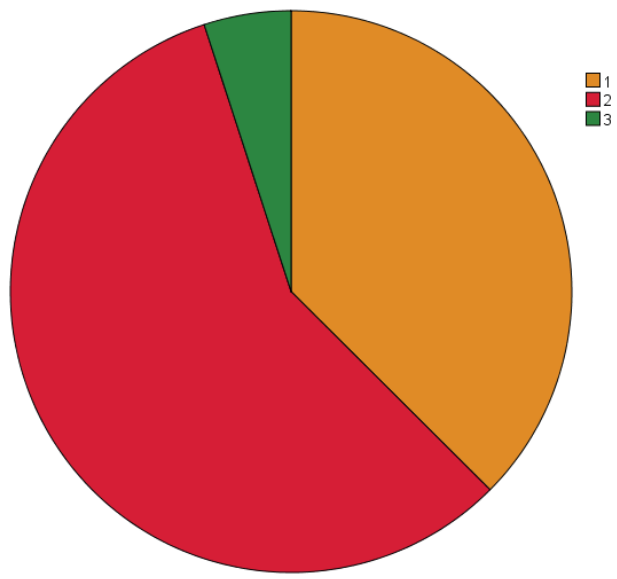

Figure 1. Pie chart showing incidence of different types of thyroid disorder.

1=Hyperthyroidism 2=Hypothyroidism 3=secondary hyperthyroidism. 
Hypothyroidism was found to be most prevalent, wherein primary hypothyroidism and subclinical hypothyroidism (not shown separately in the pie chart) together accounted for $57.5 \%$ of the total cases of thyroid disorder. There is a wealth of evidence to suggest that hypothyroidism is the most common type of thyroid disorder. Hyperthyroidism was found in $37.5 \%$ of the participants of which $21.8 \%$ had presented with clinical findings suggestive of thyrotoxicosis. Secondary hyperthyroidism was the least common type of thyroid disorder identified $(5.0 \%)$.

Next, we examined which of the variables such as age, BMI, pulse, BP, and FBS for independently predictedhypothyroidism $(\mathrm{n}=195)$, hyperthyroidism $(\mathrm{n}=127)$ and secondary hyperthyroidism $(\mathrm{n}=$ 17) by using multiple regression models in SPSS. Overall, the regression model did not statistically significantly predict hypothyroidism, $\mathrm{F}(6,188)=$ $1.31, p=0.25, R 2=0.04$. None of these variables studied added statistically significantly to the prediction of hypothyroidism. Also, the regression model did not statistically significantly predict hyperthyroidism, $\mathrm{F}(6,120)=0.99, \mathrm{p}=0.43, \mathrm{R} 2=$ 0.05 . None of the variables except BMI $(p=0.05)$ added statistically significantly to the prediction of hyperthyroidism. For secondary hyperthyroidism, although the regression model overall statistically significantly predicted the condition, $\mathrm{F}(6,16)=$ $3.40, p=0.04, \mathrm{R} 2=0.67$, none of the variables studied individually added statistically significantly to the prediction of secondary hyperthyroidism except diastolic BP $(p=0.01)$.The results suggest that measurements of BMI and diastolic BP are important parameters in predicting patients at risk of developing thyroid disorders (in particular in those with hyperthyroidism and secondary hyperthyroidism respectively) and may be considered adjunctive entities in screening for thyroid diseases.

\footnotetext{
${ }^{1}$ As female gender is widely known as an independent risk factors of thyroid disorder we did not use that variable to fit regression models.
}

\section{DISCUSSION}

In this study we examined the profile of different types of thyroid disorders encountered in patients attending a tertiary care practice in Western region of Nepal (Pokhara and surrounding places). Consistent with the literature we also found that hypothyroidism was the most prevalent thyroid disorder. In the general population various studies have shown the prevalence of hypothyroidism to be as high as $10 \%^{19-21}$. The worldwide prevalence of hypothyroidism in various studies shows a very significant variation and current prevalence ranges from $1 \%$ to $20 \%$ for sub-clinical and $1-2 \%$ for overt hypothyroidism ${ }^{24}$. Although hypothyroidism is very common endocrine problem, but frequency and severity of the symptoms vary between individuals. Sign and symptoms reflect the numerous organ systems affected by thyroid hormones but not a single clinical manifestation specially indicates thyroid dysfunction ${ }^{25}$. In our study majority of the subjects are females which indicates this is the disease of the females which is also supported by other studies ${ }^{26}$. The prevalence of hypothyroidism in our study was followed by hyperthyroidism and secondary hyperthyroidism. Nearly $87 \%$ of those with thyroid disorder were females. Using the regression models we showed that pulse and BMI are significant parameters associated with hyperthyroidism. When compared with normal participants (i.e., those without thyroid disorders) participants with thyroid disorders were found to differ significantly not just in the level of thyroid hormones and TSH, but also in age, diastolic blood pressure and pulse rates. Indeed it has been reported that that patients with thyroid disorders have abnormal pulse rates ${ }^{22}$.

Following limitations of our study should be noted; data were collected from patients attending a tertiary care hospital/centre so that our results may not be representative of the general population. Also, patients whose thyroid hormone levels were normal may have been on medication previously as a result of which their blood tests may have revealed normal T3, T4 or TSH levels, and hence were classified as normal. Also we did not examine 
life style factors such as the effect of smoking and co-morbid conditions like diabetes and depression. Smoking has been linked with thyroid disorder ${ }^{23}$. Nonetheless, our findings highlight that thyroid disorder is a significant clinical burden and a major public health concern in Western Nepal, thereby warranting a need to roll out thyroid screening programmes in the general population so that this condition can be identified and treated early. Our findings also suggest BMI which is easily measurable in the community may be useful additional tools for screening patients with thyroid disorders, particularly hyperthyroidism.

\section{REFERENCES}

1. Bernal J, Guadaño-Ferraz A, Morte B. Thyroid hormone transporters--functions and clinical implications. Nat Rev Endocrinol. 2015 Jul;11(7):406-17. doi: 10.1038/nrendo.2015.66. Epub 2015 May 5.

2. Brüning JC, Gautam D, Burks DJ, Gillette J, Schubert M, Orban PC, Klein R, Krone W, Müller-Wieland D, Kahn CR. Role of brain insulin receptor in control of body weight and reproduction. Science. 2000 Sep 22;289(5487):2122-5.Doi: 10.1126/ science.289.5487.2122.

3. British Thyroid Foundation. Your guide to thyroid disorder. 2019.

4. Taylor PN, Albrecht D, Scholz A, GutierrezBuey G, Lazarus JH, Dayan CM, Okosieme OE. Global epidemiology of hyperthyroidism and hypothyroidism. Nat Rev Endocrinol. 2018 May;14(5):301-316. doi: 10.1038/ nrendo.2018.18.

5. Gaitonde DY, Rowley KD, Sweeney LB. Hypothyroidism: an update. Am Fam Physician. 2012 Aug 1;86(3):244-51.

6. Caturegli P, De Remigis A, Rose NR. Hashimoto thyroiditis: clinical and diagnostic criteria. Autoimmun Rev. 2014 Apr-May;13(4-5):391-7. doi: 10.1016/j.autrev.2014.01.007.

7. Vanderpump MP. The epidemiology of thyroid disease. Br Med Bull. 2011;99:39-51. doi: 10.1093/bmb/ldr030.

8. Peltzer K, Pengpid S. Anticipated stigma in chronic illness patients in Cambodia, Myanmar and Vietnam. Nagoya J Med Sci. 2016 Dec; 78(4): 423-435. doi: 10.18999/ nagjms.78.4.423.

9. Walsh JP. Managing thyroid disease in general practice. Med. J. Aust. 205, 179-184 (2016). Doi: $10.5694 / \mathrm{mja1} 6.00545$.

10. Hollowell, J. G. et al. Serum TSH, T(4), and thyroid antibodies in the United States population (1988 to 1994): National Health and Nutrition Examination Survey (NHANES III). J. Clin. Endocrinol. Metab. 87, 489-499 (2002). Doi: $10.1210 /$ jcem.87.2.8182.

11. ArmendiaMadariaga A, Santos Palacios S., Guillen-Grima F. Galofre JC. The incidence and prevalence of thyroid dysfunction in Europe: a meta-analysis. J. Clin. Endocrinol. Metab. 99, 923-931 (2014).Doi: 10.1210/jc.2013-2409.

12. Vanderpump MP, Tunbridge WM, French JM, Appleton D, Bates D, Clark F, Grimley Evans J, Hasan DM, Rodgers H, Tunbridge Fet al. The incidence of thyroid disorders in the community: a twenty-year follow-up of the Whickham Survey. Clin. Endocrinol. 43, 55-68 (1995).Doi: 10.1111/j.1365-2265.1995. tb01894.x.

13. Laurberg P, Jørgensen T, Perrild H, Ovesen L, Knudsen N, Pedersen IB, Rasmussen LB, Carlé A, Vejbjerg P. The Danish investigation on iodine intake and thyroid disease,DanThyr: status and perspectives. Eur. J. Endocrinol. 155, 219-228 (2006).Doi: 10.1530/eje.1.02210.

14. Gupta p, Agrawal PKB, Gauchan B. Prevalence of thyroid disorder in a primary care district hospital of Nepal. Journal of Nepal Medical Association. 1 57, 216 (2019).Doi: 10.31729/ jnma.4240.

15. Chaudhary LN, Khatiwada S, Gelal B, Gautam S, Lamsal M, Pokharel H, Baral N. Iodine and Thyroid Function Status, and Anti-thyroid Peroxidase Antibody among Pregnant Women in Eastern Nepal. J Nepal Health Res Counc. 2017 Sep 8;15(2):114-119.

16. Khatiwada S, Rajendra KC, Gautam S, LamsalM, Baral N. Thyroid dysfunction and dyslipidemia in chronic kidney disease patients. 
BMC EndocrDisord. 2015 Oct 29;15:65. Doi: 10.1186/s12902-015-0063-9.

17. Tamang MK, Gelal B, Tamang B, Lamsal M, Brodie D, Baral N. Excess urinary iodine concentration and thyroid dysfunction among school age children of eastern Nepal: a matter of concern. BMC Res Notes. 2019 May 27;12(1):294. Doi: 10.1186/s13104-0194332-y.

18. von Elm E, Altman DG, Egger M, et al. The strengthening the reporting of observational studies in epidemiology (STROBE) statement: guidelines for reporting observational studies. Int J Surg2014;12:1495-9.Doi: 10.1016/j. ijsu.2014.07.013.

19. Klein I, Danzi S. Thyroid disease and the heart. Circulation. 2007. October 9; 116 15: 1725-35. Doi: 10.1161/ CIRCULATIONAHA.106.678326.

20. Ochs N, Auer R, Bauer DC, Nanchen D, Gussekloo J, Cornuz J, Rodondi N. Metaanalysis: subclinical thyroid dysfunction and the risk for coronary heart disease and mortality. Ann Intern Med. 2008. June 3; 148 11: 832-45. Doi: 10.7326/0003-4819-148-11-20080603000225 .
21. Razvi S, Shakoor A, Vanderpump M, Weaver JU, Pearce SH. The influence of age on the relationship between subclinical hypothyroidism and ischemic heart disease: a meta-analysis. J ClinEndocrinolMetab. 2008. August; 93 8: 2998-3007. Doi: 10.1210/ jc.2008-0167.

22. Udovcic M, Pena RH, Patham B, Tabatabai L, Kansara A. Hypothyroidism and the Heart. Methodist DebakeyCardiovasc J. 2017 AprJun; 13(2): 55-59. doi: 10.14797/mdcj-13-255.

23. Wiersinga WM. Smoking and thyroid. ClinEndocrinol (Oxf). 2013 Aug;79(2):145-51. doi: 10.1111/cen.12222. Epub 2013 May 11. 